\title{
Differential expression of miRNAs in the presence of B chromosome in the cichlid fish Astatotilapia latifasciata
}

Jordana Inácio Nascimento-Oliveira' ${ }^{1}$, Bruno Evaristo Almeida Fantinatti ${ }^{2}$, Ivan Rodrigo Wolf ${ }^{1}$, Adauto Lima Cardoso ${ }^{1}$, Erica Ramos ${ }^{1}$, Nathalie Rieder ${ }^{3}$, Rogerio de Oliveira ${ }^{4}$ and Cesar Martins ${ }^{1 *}$

\begin{abstract}
Background: B chromosomes (Bs) are extra elements observed in diverse eukaryotes, including animals, plants and fungi. Although Bs were first identified a century ago and have been studied in hundreds of species, their biology is still enigmatic. Recent advances in omics and big data technologies are revolutionizing the B biology field. These advances allow analyses of DNA, RNA, proteins and the construction of interactive networks for understanding the B composition and behavior in the cell. Several genes have been detected on the B chromosomes, although the interaction of $B$ sequences and the normal genome remains poorly understood.

Results: We identified 727 miRNA precursors in the A. latifasciata genome, 66\% which were novel predicted sequences that had not been identified before. We were able to report the A. latifasciata-specific miRNAs and common miRNAs identified in other fish species. For the samples carrying the $B$ chromosome $\left(B^{+}\right)$, we identified 104 differentially expressed (DE) miRNAs that are down or upregulated compared to samples without $B$ chromosome $\left(B^{-}\right)(p<0.05)$. These miRNAs share common targets in the brain, muscle and gonads. These targets were used to construct a protein-protein-miRNA network showing the high interaction between the targets of differentially expressed miRNAs in the $\mathrm{B}^{+}$chromosome samples. Among the DE-miRNA targets there are proteincoding genes reported for the B chromosome that are present in the protein-protein-miRNA network. Additionally, Gene Ontology (GO) terms related to nuclear matrix organization and response to stimulus are exclusive to DE miRNA targets of $\mathrm{B}^{+}$samples.
\end{abstract}

Conclusions: This study is the first to report the connection of B chromosomes and miRNAs in a vertebrate species. We observed that the B chromosome impacts the miRNAs expression in several tissues and these miRNAs target several mRNAs involved with important biological processes.

Keywords: RNA-seq, Supernumerary chromosome, Selfish element, Genomic, Transcriptome, Small noncoding RNAs, Cichlid, Fish, Teleost

\footnotetext{
*Correspondence: cesar.martins@unesp.br

${ }^{1}$ Department of Structural and Functional Biology, Institute of Bioscience at Botucatu, São Paulo State University (UNESP), Botucatu, SP 18618-689, Brazil

Full list of author information is available at the end of the article
} 


\section{Background}

B chromosomes (Bs) are extra and nonessential elements found in approximately 10 to $15 \%$ of karyotyped organisms, ranging from fungi to plants and animals, and do not follow classical Mendelian inheritance patterns [15]. The origin, evolution, genome content and morphology of B chromosomes vary among organisms [6].

A proto- $B$ chromosome can emerge from chromosomal rearrangements, partial duplication of A chromosomes (normal chromosomes of the karyotype) or nonmeiotic disjunction [5]. This new element increases its genomic content by insertion of A chromosome sequences copies, including various repetitive DNA classes [7-9], protein-coding genes [2, 10-13], pseudogenes [14], retrogenes [15], organellar DNA sequences [16] and noncoding sequences [17-20]. Duplicated sequences in $\mathrm{B}$ chromosomes have been proposed to facilitate its permanence in the host genome. These sequences may help the $\mathrm{B}$ chromosome drive during gametogenesis avoiding $\mathrm{B}$ elimination $[5,11]$. In this way, the characterization of the $\mathrm{B}$ genomic content and its effects using genomics and bioinformatics tools is a promising approach to understand this extra element and its relation to the host genome [21, 22].

Regarding to noncoding RNAs, some few sequences have already been reported either in the B chromosome or impacting in the expression of autosomal sequences in the $\mathrm{B}^{+}$samples. Noncoding RNAs exert strong effects on cell biological processes and are potentially related to the presence of the $B$ chromosome $[18,19,23]$. Among noncoding RNAs, microRNAs (miRNAs) ( 22 nucleotides long) act in the translation control by promoting the degradation or cleavage of mRNAs. That is why they are responsible for the control of important processes, such as development and differentiation, cell cycle regulation, stress and aging, and some diseases such as cancer. Notably, miRNAs are one of the most abundant regulators in the genome and several of them are highly conserved among organisms [24-26].

The biogenesis of miRNAs starts in the nucleus with the transcription of the primary miRNA (pri-miRNA) that has a hairpin structure and is processed to form a RNA duplex, named pre-miRNA. The pre-miRNA is transported to the cytoplasm and processed by the Dicer, that cleaves the RNA duplex into two single RNA molecules, $5 p$ and $3 p$ arms. Only one arm will become the mature sequence while the another one will be degraded [27]. When associated with the Argonaute protein, the mature miRNA interacts with its target based on antisense Watson-Crick pairing that occurs mainly in the 3' untranslated regions (3'UTR) of mRNAs [28].

The connection between $\mathrm{B}$ chromosome and miRNAs has only been investigated in two organisms, an invertebrate and a plant species. The wasp Nasonia vitripennis carries a selfish supernumerary chromosome called PSR (Paternal Sex Ratio) that transcribes several small RNAs sequences, such as microRNAs, small interference RNAs and PIWI-Interacting RNAs [18]. In maize, B-derived miRNAs were found to affect A chromosome miRNA expression [23]. However, to the best of our knowledge, these reports are the only two describing small noncoding RNA sequences in the context of B chromosomes. Thus, the impact of B chromosome duplications in the small noncoding RNAs expression is poorly understood.

Among vertebrates, $\mathrm{B}$ chromosomes have already been described in approximately 100 fish species [4], corresponding to $16.28 \%$ of karyotyped species [29]. Teleost fishes are important for evolutionary studies, especially in the Cichlidae family, due to their rapid adaptive radiation in East African great lakes [30-32]. B chromosomes were detected in several cichlid species [30, 33, 34]. Among them, the African cichlid Astatotilapia latifasciata, which carries one or two B chromosomes in both sexes, has been extensively investigated through classical cytogenetics [33, 35], molecular biology $[9,36]$ and, more recently, genomic approaches [11, 15, 19, 37]. Repetitive elements [9], coding genes [11] and a long noncoding RNA [19] have already been identified in the B chromosome of A. latifasciata. Some of these sequences revealed a differential expression in the $\mathrm{B}^{+}$samples suggesting transcription activity and involvement of this extra element into several biological pathways [19, 20, 38].

The A. latifasciata B chromosome content has been investigated by comparing sequencing from $\mathrm{B}^{-}$and $\mathrm{B}^{+}$ DNA and RNA samples. The B chromosome gene content was first reported through genomic coverage rate analysis based on Illumina high coverage sequencing and 454 Life Sciences sequencing of a microdissected B chromosome [11]. In this work, the microdissected B chromosome sequences were compared to Metriaclima zebra reference genome, and the first B-genes of $A$. latifasciata were reported. Later, the A. latifasciata draft genome was constructed using Illumina high scale data and identified several duplicated contigs in the $\mathrm{B}$ chromosome [37]. The coverage ratio compares coverage of sequenced among $\mathrm{B}^{-}$and $\mathrm{B}^{+}$samples aligned against a reference assembled genome, which allows to identify higher coverage regions on the $\mathrm{B}^{+}$sequencing dataset, that represent duplicated regions in the $B$ chromosome [11, 39].

In this study, large-scale small RNA sequences (sRNAseq) were generated from the brain, muscle and gonads of $\mathrm{B}^{-}$and $\mathrm{B}^{+}$individuals of both sexes of A. latifasciata. Using bioinformatics approaches, the A. latifasciata miRNA profile was described, and compared with other teleost miRNAs, mainly cichlid species. This allowed the identification of conserved and specific novel miRNAs. 
In this work, we introduce the application of several bioinformatics tools to investigate miRNA sequences in the context of B chromosomes based on coverage ratio analysis and the generated "B-blocks" as previously reported [11]. B-blocks are putative genomic regions observed on B chromosomes and detected via coverage ratio analysis as a result of NGS read coverage comparison between the two sequenced genomic datasets $\left(\mathrm{B}^{+}\right.$and $\left.\mathrm{B}^{-}\right)$. We described 104 miRNAs that were differentially expressed (DE), either up or downregulated in the presence of the B chromosome compared to samples without B chromosome (the control). These miRNAs have common mRNA targets in the brain, muscle and gonads. Additionally, we found protein coding genes already described in the $\mathrm{B}$ chromosome (the B genes) as targets of DE miRNAs. Moreover, a network based on human protein-protein interactions of the DE miRNAs targets highlights the great potential of DE miRNAs in the influence of B chromosomes over several biological processes.

By combining the sRNAseq with the availability of $A$. latifasciata genomic and mRNA transcriptomic data we described the miRNome of this cichlid species. Further, this is the first study that relates the miRNA expression and the $\mathrm{B}$ chromosome presence in a vertebrate species. This is also the first report of coding and noncoding interactions related to $\mathrm{B}$ chromosome presence.

\section{Results}

\section{The A. latifasciata miRNome}

A nonredundant dataset was constructed based on miRBase fish miRNAs to create a miRNA reference list (see the Materials and Methods). The procedure resulted in 1456 precursors and 1234 mature sequences. This fish miRNA reference list was used to identify the miRNAs in the sRNA-seq data and annotate them in the A. latifasciata genome. We identified 727 miRNA precursors (pre-miRNAs) throughout the A. latifasciata genome (see Additional File 1). Among them, 246 (33.84\%) premiRNAs have similarity with described miRNAs in miRBase, and they are called known miRNAs. On the other hand, sequences that were not similar to existing miRNAs are called novel. Additionally, novel miRNAs could only present seed similarity to existing miRNAs, indicating new miRNAs probably belonging to an existent miRNA family [40]. The novel $A$. latifasciata premiRNAs represent 481 (66.16\%) precursor sequences, and $29.31 \%$ of them exhibit miRBase seed similarity (nucleotides 2-8 from the $5^{\prime}$ end of the mature miRNA).

Clustered miRNAs can be arranged in a 5 kilobase genomic region long and generally are related to the same transcription factors [39]. Here, we considered clustered miRNAs when the sequences were found in the same genomic contig and not exceeded 5 kilobases distance. The clustered miRNAs (pre-miRNAs on the same genome contig) accounted for 232 (31.91\%) sequences; the longest cluster contained 9 pre-miRNAs. Also, 495 (36.31\%) precursor sequences are single miRNAs in a genomic contig. Usually, one arm (5p or 3p) is highly expressed in the cell, while the other arm can be degraded [27]. Thus, comparing the expression of the arms in the sRNAseq, $55 \%$ of premiRNAs displayed higher expression in the $5 \mathrm{p}$ arm mature sequence. Transcription was identified on the minus strand for $368(50.62 \%)$ pre-miRNAs and on the plus strand for 359 (49.38\%) pre-miRNAs. All the results mentioned above are described in the Fig. 1a and b.

The last miRBase release added seven new fish species and 2050 new sequences, summing up 3687 miRNAs sequences (Additional File 11 - Table S3). From these new species in the last release, five are cichlids and contribute to 1300 miRNAs sequences. The miRNA seed sequences from $A$. latifasciata have similarity with 15 fish species (representatives of nine teleost families) in miRBase, corresponding to 246 pre-miRNAs (Additional File 1). The species were verified according to the three first letters of the miRNA ID, which corresponds to the species ID in the animal miRNA nomenclature pattern, as indicated next in the species name. Thirtyfive percent of known miRNA seed sequences share similarity with cichlids (Astatotilapia burtoni - abu, Metriaclima zebra - mze, Neolamprologus brichardi nbr, Oreochromis niloticus - oni, and Pundamilia nyererei - pny); $65 \%$ show similarity with other teleost families (one species of Adrianichthyidae, Oryzias latipes ola; two species of the Cyprinidae family, Cyprinus carpio - ccr, and Danio rerio, - dre; one species of Gadidae, Gadus morhua - gmo; one species of Ictaluridae, Ictalurus punctatus - ipu; one species of Pleuronectidae, Hippoglossus hippoglossus - hhi; one species of Salmonidae, Salmo salar - ssa; and two species of Tetraodontidae, Fugu rubripes - fru, and Tetraodon nigroviridis tni) (Fig. 1b and c). Even not being the most representative reference species Danio rerio seeds are well represented among the known miRNAs, probably indicating the presence of highly conserved miRNAs in the A. latifasciata miRNome (Additional File 9 - Table S3).

Approximately $70 \%$ of novel miRNAs are not similar to any seed from miRBase, indicating their potential as either new specific or nondescribed miRNAs (Fig. $1 \mathrm{~b}$ and c). When considering only the novel miRNAs that present seed similarity from miRBase, $60 \%$ are similar to seed sequences from $A$. burtoni, M. zebra and O. niloticus, which probably represent exclusive conserved miRNAs families among cichlids (absent or not conserved in other groups).

\section{Searching for miRNA genes on the B chromosome}

We performed three different strategies in order to investigate the miRNA presence in the B chromosome. All 


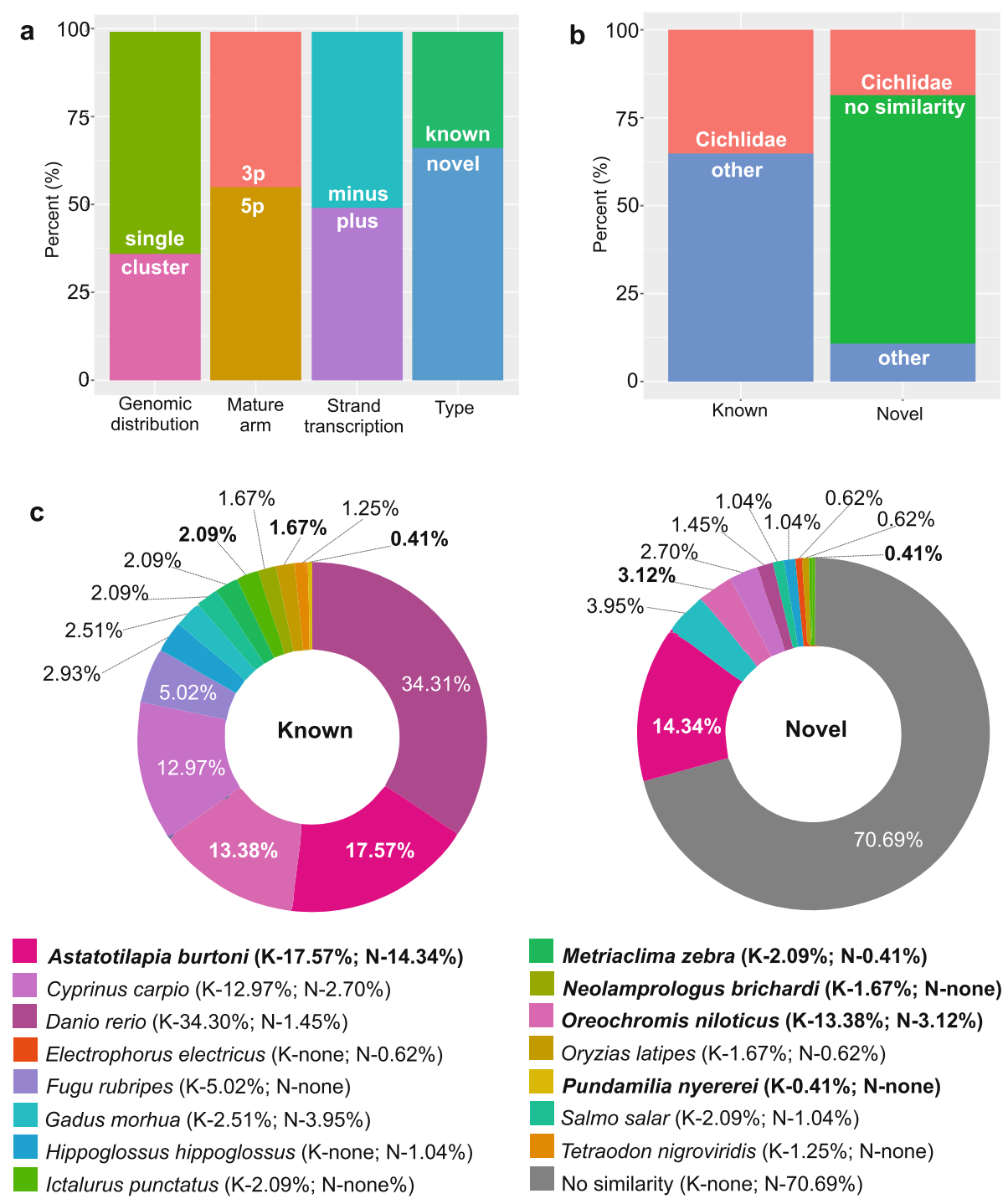

Fig. 1 Description of the A. latifasciata miRNome. a Percentage of miRNA characteristics. Genomic distribution: the miRNA precursor arrangement on the genome; Mature arm: the mature sequence with more reads on RNA-Seq; Strand transcription: precursor transcription strand; Type: if the miRNA is similar to another known miRNA from miRBase (known) or if is probably a new miRNA sequence (novel). $\mathbf{b}$ Percentage of similarity with cichlids and other fishes. c Percentage of miRNA similarity compared with fish sequences from miRBase. Cichlid species are highlighted in bold. K, Known miRNAs; N, novel miRNAs

strategies were based on DNA and sRNAseq comparison of $\mathrm{B}^{-}$and $\mathrm{B}^{+}$samples (see Material and Methods).

The coverage ratio analysis (first strategy) consists in screening the coverage difference between the $\mathrm{B}^{-}$and $\mathrm{B}^{+}$ genomic reads aligned against the A. latifasciata assembled genome. This strategy allows us to find segments of A chromosomes that are duplicated on the B chromosome. We did not detect any miRNA gene inside a genomic region with coverage corresponding to the $\mathrm{B}$ chromosome ( $\mathrm{B}^{+}$blocks). The second method was based on alignments using the sRNAseq reads from $\mathrm{B}^{-}$and $\mathrm{B}^{+}$ samples of all tissues that failed to align in the A. latifasciata reference genome. Then, we performed a second alignment using these unaligned sRNAseq reads to $\mathrm{B}^{+}$ assembled genome (the A. latifasciata DNA with B chromosome). This method identified 21 novel miRNA genes, of which 6 were exclusively expressed in $\mathrm{B}^{+}$samples (Additional File 2 and Additional File 3). Thus, ten $\mathrm{B}^{+}$assembly miRNA genes were selected for validation, as they had an adequate length for qPCR primer construction. Only one miRNA gene (called here novel 2026- $\mathrm{B}^{+}$, Fig. 2a, Additional File 3) located in contig NODE_313069 from the $\mathrm{B}^{+}$assembly was PCR-amplified (Additional File 4). However, amplification was observed in both $\mathrm{B}^{-}$and $\mathrm{B}^{+}$samples (data not shown). qPCR experiments were performed to confirm that this genomic 

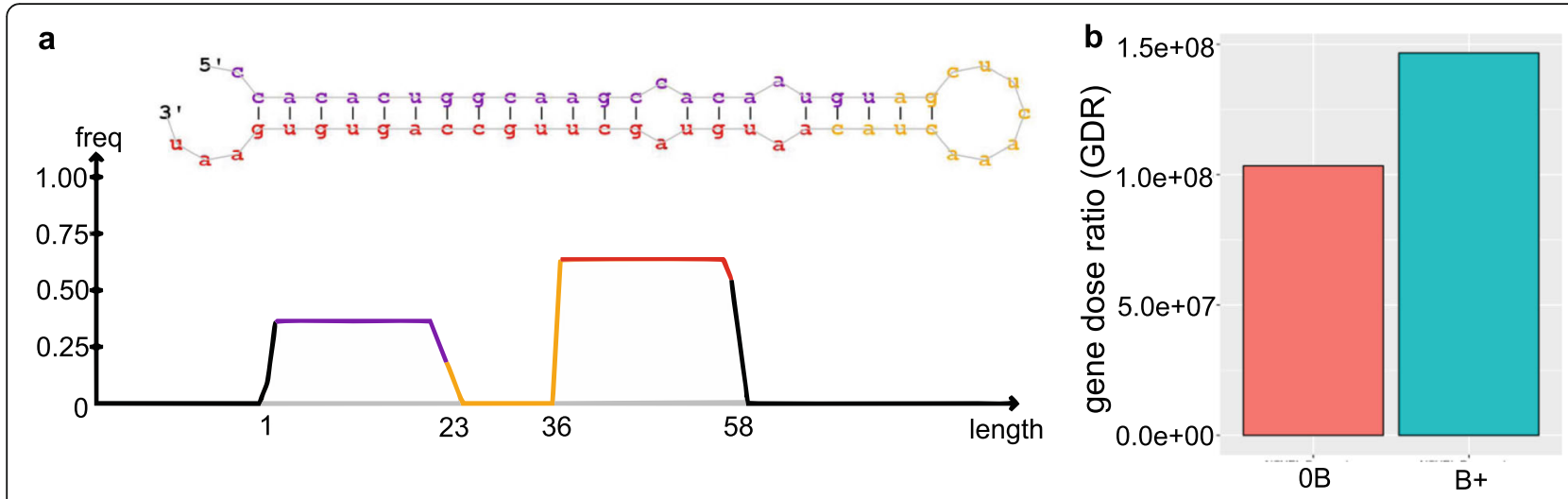

C

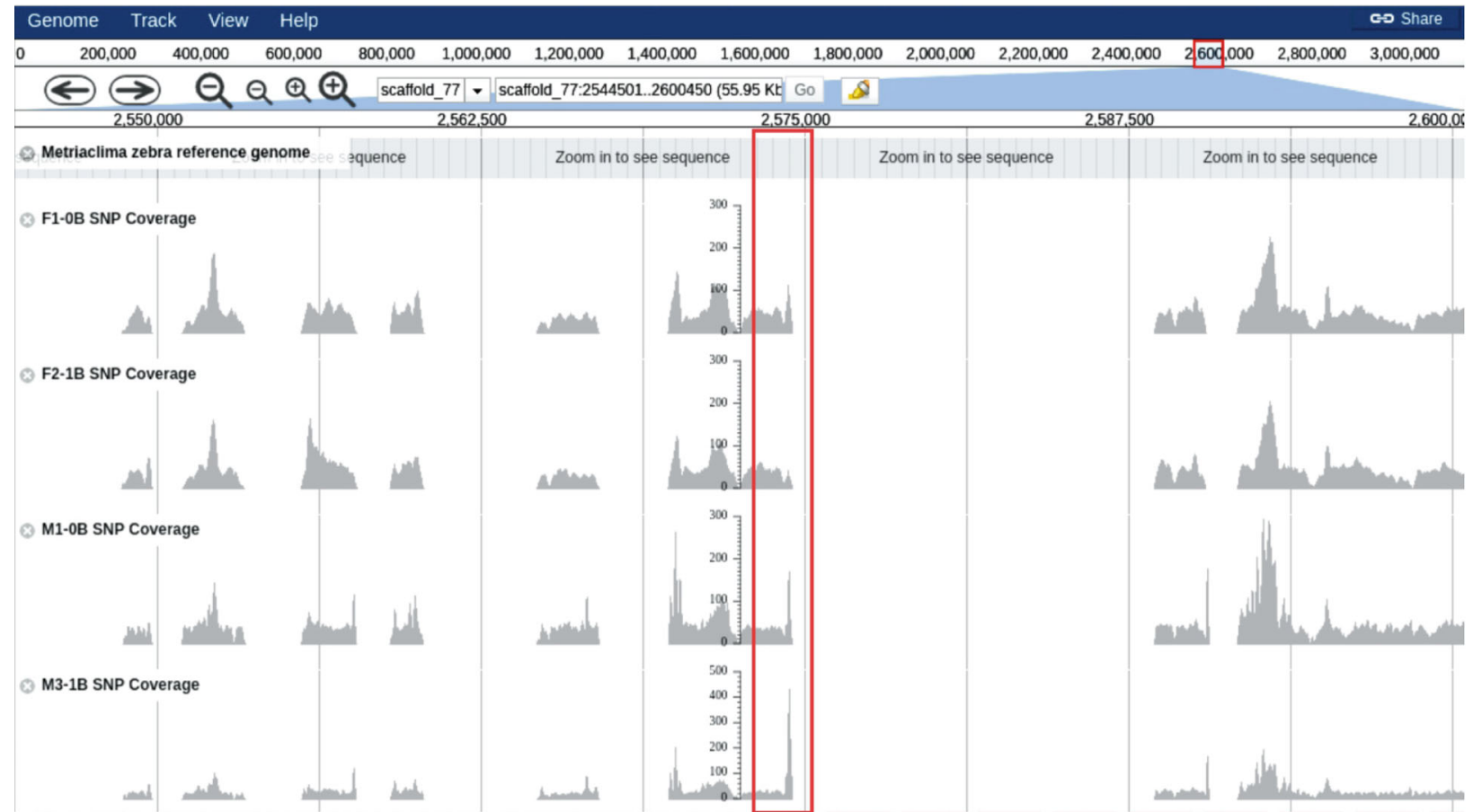

Fig. 2 Functional miRNA absence in the $B^{+}$genome assembly. a The novel_2026- $B^{+}$predicted from the $B^{+}$assembly. This miRNA has a stemloop secondary structure. $\mathbf{b}$ The NODE_313069 qPCR for $\mathrm{B}^{-}$and $\mathrm{B}^{+}$DNA samples. The difference between $\mathrm{B}^{-}$and $\mathrm{B}^{+}$amplification was not significant ( $p$-value $13>0.05$ ). $\mathbf{c}$ Predicted novel_2026- $\mathrm{B}^{+}$match with $M$. zebra scafold_77. The A. latifasciada genomic sequencing reads are shown below the M. zebra reference genome, F1-0B SNP coverage (female $B^{-}$sample), F-1B SNP coverage (female $B^{+}$sample), M1-0B SNP coverage (male $\mathrm{B}^{-}$sample), M3-1B SNP coverage (male $\mathrm{B}^{+}$sample). The gray area is the read coverage, the blank spaces show no aligned reads in this region, and the red rectangle highlights the novel_2026-B+ NODE_313069 region matching the M. zebra assembly

segment was in both groups of individuals $\left(\mathrm{B}^{-}\right.$and $\left.\mathrm{B}^{+}\right)$. The gene dose ratio (GDR) compares the relative gene copies trough qPCR, which showed that the novel_2026$\mathrm{B}^{+}$region has not GDR difference in the $\mathrm{B}^{-}$and $\mathrm{B}^{+}$genomes, meaning the same number of copies in both genomes (Fig. 2b). Additionally, novel_2026- $\mathrm{B}^{+}$is similar to scaffold_77 of $M$. zebra, where $\mathrm{B}^{-}$and $\mathrm{B}^{+}$genomic reads are aligned (Fig. 2c). The $A$. latifasciata $\mathrm{B}^{-}$and $\mathrm{B}^{+}$ genomes have several assembly gaps that might justify the absence of miRNA alignments. The evidence suggests that novel_2026- $\mathrm{B}^{+}$occurs in the A. latifasciata genome but is not present in the $\mathrm{B}$ chromosome. The gaps are probably resulting of an assembly bias in the $A$. latifasciata genome, where the short Illumina reads caused the region to be ignored during the assembly (Additional File 4 - Figure S1).

The third approach was predicting miRNAs using the miRBase reference and the sRNAseq, as the previous strategies, but set the genomic "B-blocks" filtered by Jehangir et al. [37] as the reference background. With this strategy we would like to confirm if no duplicated miRNAs were missed in our manual coverage ratio 
strategy (first strategy). This prediction found 33 premiRNAs on the B-blocks. Two miRNAs are similar to mir-2188, and the others are novel (no miRBase similarity) (Additional File 6). These 33 miRNAs did not show interaction with any mRNAs and were not considered for further analysis.

Therefore, based on the results obtained with these three strategies, we did not find strong evidences of miRNAs in the B chromosome. We discuss the limitations further.

\section{Effects of B chromosomes on miRNA expression}

The differential expression analysis was performed by comparing $\mathrm{B}^{+}$samples against the samples without $\mathrm{B}$ chromosome (as the control) in each tissue (brain, gonads and muscle) and each sex (male and female). Several miRNAs were differentially expressed $(D E)$ in $\mathrm{B}^{+}$ samples (either up or downregulated), which is why these sequences were called B DE miRNAs (B-DE-miRNAs). The heatmap in the Fig. 3a shows the upregulated sequences (positive FoldChange in green gradient) and downregulated sequences (negative FoldChange in red gradient) in $\mathrm{B}^{+}$samples in each sex and tissue. The profile of DE miRNAs between tissues, sexes and the presence of the $\mathrm{B}$ chromosome detected 104 nonredundant miRNAs (Fig. 3a and b). In brain, 12 novel and 2 known B-DE-miRNAs were found in females and 12 novel and 7 known B-DEmiRNAs were detected in males. In gonads, 29 novel and 18 known B-DE-miRNAs were found in females and 8 novel and 3 known B-DE-miRNAs were detected in males. In muscle, 6 novel and 4 known BDE-miRNAs were found in females and 8 novel and 4 known B-DE-miRNAs were detected in males.

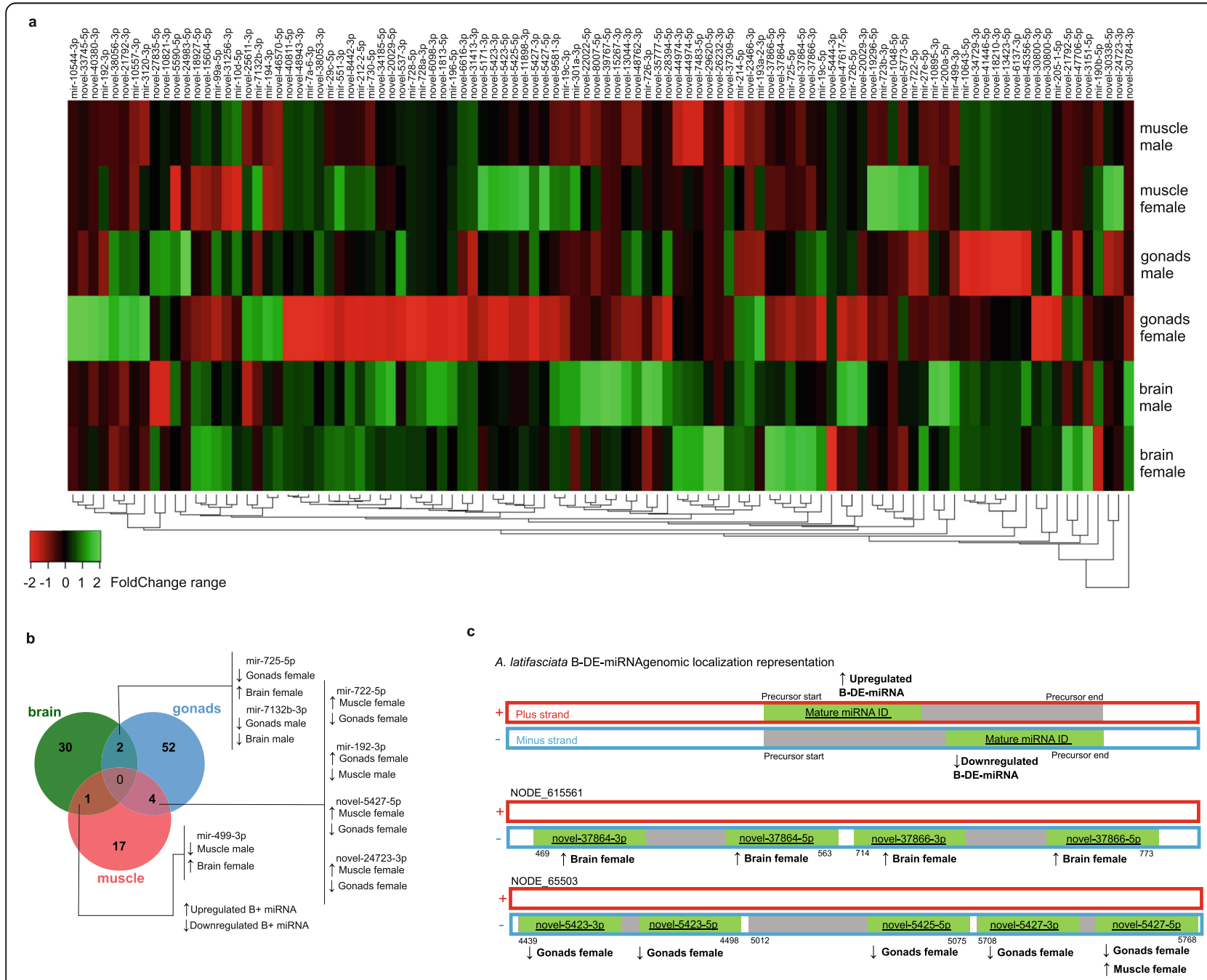

Fig. 3 Differential expression analysis. a Nonredundant differentially expressed miRNAs in $\mathrm{B}^{+}$samples (green represents upregulated miRNAs and red represents downregulated, considering $p<0.05$ to $>1.5$ fold change). $\mathbf{b}$ Venn diagram of DE miRNAs among tissues. $\mathbf{c}$ Representation of two clustered DE-B-miRNAs structural organization in genomic contigs 
Table 1 Clustered miRNAs composed by downregulated $(\downarrow)$ and upregulated $(\uparrow)$ B-DE-miRNAs in brain (BR), gonad (G), muscle (MU), female (F), male (M). FG, FoldChange

\begin{tabular}{|c|c|c|c|c|c|c|c|}
\hline Contig & $\begin{array}{l}\text { Pre-miRNA } \\
\text { ID }\end{array}$ & $\begin{array}{l}\text { DNA } \\
\text { strand }\end{array}$ & $\begin{array}{l}\text { Pre-miRNA start- } \\
\text { end }\end{array}$ & Seed similarity & $\begin{array}{l}\text { Mature B-DE- } \\
\text { miRNA }\end{array}$ & $\begin{array}{l}\text { Expression in } \mathrm{B}^{+} \\
\text {samples }\end{array}$ & $\begin{array}{l}F C \text { in } B^{+} \\
\text {sampes }\end{array}$ \\
\hline \multirow{2}{*}{$\begin{array}{l}\mathrm{NODE}_{-} \\
173406\end{array}$} & mir-99a & - & $9625-9682$ & nbr-mir-99a & mir-99a-5p & $\downarrow$ GO_F & -1.23 \\
\hline & novel_13044 & + & $9626-9684$ & $\begin{array}{l}\text { gmo-mir-100b- } \\
5 p\end{array}$ & novel_13044-3p & $\uparrow B R \_M$ & +1.38 \\
\hline \multirow{4}{*}{$\begin{array}{l}\mathrm{NODE}_{-} \\
615561\end{array}$} & novel_37864 & - & 499-563 & abu-mir-27c & novel_37864-5p & $\uparrow B R \_F$ & +2.78 \\
\hline & & & & & novel_37864-3p & $\uparrow B R_{-} F$ & +2.49 \\
\hline & novel_37866 & - & 714-773 & abu-mir-23c & novel_37864-5p & $\uparrow B R \_F$ & +2.68 \\
\hline & & & & & novel_37864-3p & $\uparrow B R_{-} F$ & +2.51 \\
\hline \multirow[t]{6}{*}{ NODE_65503 } & novel_5423 & - & 4439-4498 & ccr-mir-217 & novel_5423-5p & $\downarrow$ GO_F & -2.16 \\
\hline & & & & & novel_5423-3p & $\downarrow$ GO_F & -1.76 \\
\hline & novel_5425 & - & $5012-5075$ & ccr-mir-216a & novel_5425-5p & $\downarrow$ GO_F & -1.11 \\
\hline & novel_5427 & - & $5708-5768$ & $c c r-m i r-216 b$ & novel_5427-3p & $\downarrow$ GO_F & -2.19 \\
\hline & & & & & novel_5427-5p & $\downarrow$ GO_F & -1.70 \\
\hline & & & & & & $\uparrow M U \_F$ & +2.50 \\
\hline \multirow{2}{*}{$\begin{array}{l}\mathrm{NODE}_{-} \\
843581\end{array}$} & mir-194 & - & $58381-58436$ & ccr-mir-194 & mir-194-3p & $\downarrow M U \_M$ & -2.33 \\
\hline & mir-192 & - & $58185-58246$ & ccr-mir-192 & mir-192-3p & $\downarrow M U \_M$ & -2.67 \\
\hline \multirow[t]{2}{*}{ NODE_91705 } & mir-212-2 & - & $2623-2690$ & dre-mir-212-2 & mir-212-2-5p & $\downarrow$ GO_F & -1.89 \\
\hline & novel_7483 & - & $4415-4473$ & gmo-mir-2184 & novel_7483-5p & $\uparrow B R \_F$ & +1.27 \\
\hline
\end{tabular}

Five genomic regions carry B-DE-miRNAs, forming clustered miRNAs (Fig. $3 \mathrm{c}$ and Table 1). The B-DE-miRNAs of the same cluster displayed the same DE pattern in a particular tissue. However, a cluster can have different expression profiles among tissues. Thus, a unique pattern for all tissues was not identified. Considering the genomic contigs, the contig NODE_615561 contained 4 novel mature B-DEmiRNAs that were upregulated in the brains of females. These miRNAs belong to 2 novel pre-miRNAs (novel-37864 and novel-37866) that are similar to mir-27c and mir-23c from A. burtoni seeds. In contig NODE_65503, 3 novel miRNA precursors (novel-5423, novel-5425 and novel 37866) with 5 downregulated B-DE-miRNAs were detected in the gonads of females, which are similar to the mir-217 seeds from C. carpio and the mir-216a and mir-216b seeds from A. burtoni. However, the cluster on contig NODE_ 65503 also contained a mature B-DE-miRNA that was upregulated in the muscle of females; therefore, a cluster shows DE profiles according to the tissue (Fig. $3 c$ and Table 1). The distance between the pre-miRNAs genes is described in the start and end of pre-miRNAs sequences on Table 1. Although each tissue has a different miRNA expression profile, no difference in drosha and dicer gene expression was observed in $\mathrm{B}^{+}$samples (Additional File 5).

\section{The miRNA $3^{\prime} U T R$ interaction and protein-protein interaction (PPI) network}

The 3'UTR miRNA binding site was chosen to predict the A. latifasciata miRNA targets to restrict and avoid false positives results [41]. Other software were tested to miRNA:mRNA prediction, but they showed a huge number of interactions, being a problem to the filtering. The miRNA:mRNA interactions were predicted (Additional File 7) based on the miRNAs and 3'UTRs from the $A$. latifasciata transcriptome. Based on the best scores $(<-0.2$, following the software developer recommendations [42], 2,080,942 interactions were identified in the brain, 2,061,604 in gonads and 2,016,807 in muscle.

The miRNA:3'UTR interactions detected for the BDE-miRNAs formed a list composed of B-DEmiRNAs and targets (mRNAs). The protein annotations of these transcripts (Additional File 8) from each tissue are presented in a Venn diagram, and 960 proteins were shared among all compared groups (defined as B-related proteins) (Fig. 4a). The proteinprotein interaction (PPI) network from Biogrid was downloaded to evaluate whether B-related proteins had functional interactions (Additional File 9). The PPI indicates the physical and high specific contact of two or more proteins. Due the lack of fish proteinprotein interaction data base, the interactions were filtered based on the human set. As reported in several studies, there are several miRNA targets conserved among organisms [43, 44]. In this way, our extrapolation report mostly conserved interactions that could be confirmed by presence in the data bases, as the online TargetScan [42]. 


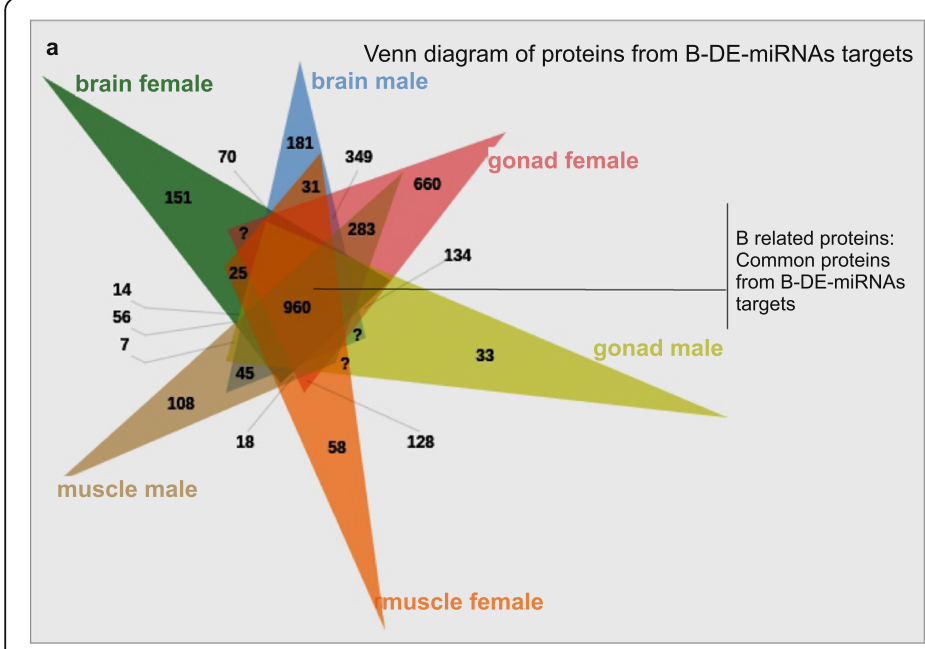

c

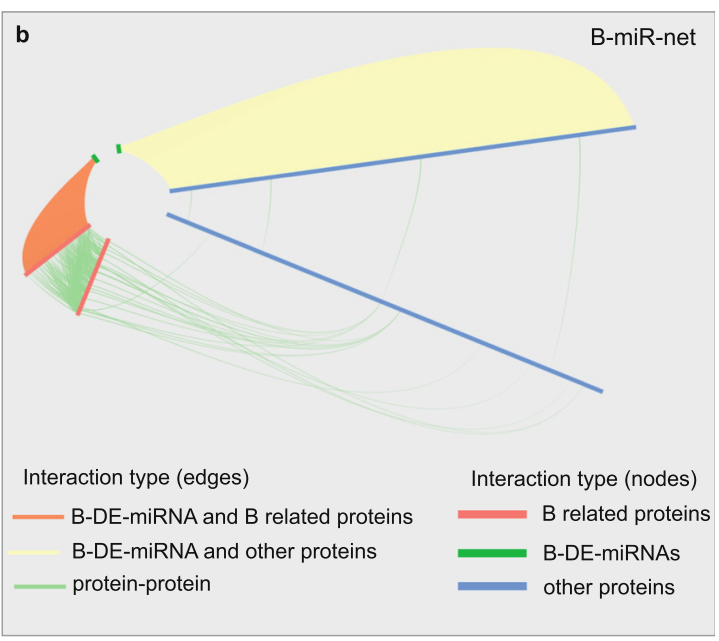

Biological process (BP) GO terms from proteins in the B-miR-net

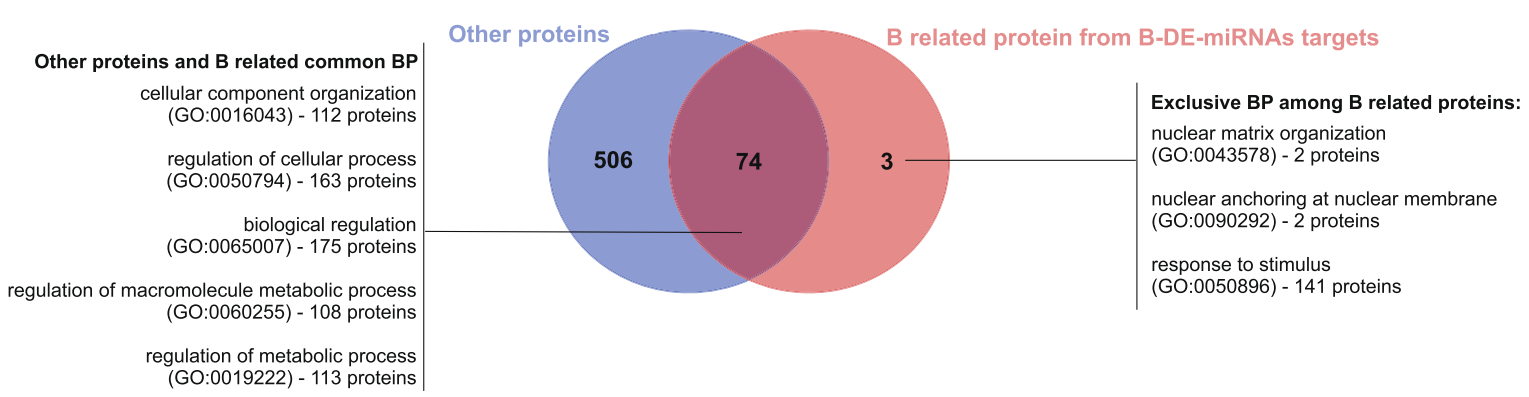

Fig. 4 The B-mir-net. a Venn diagram of common protein annotation from B-DE-miRNAs targets. $\mathbf{b}$ The network axis represents the nodes ordered by degree (from low to high beginning from the center), and edges represent the connection between the axis nodes. Axes are duplicated to show the interconnections between its own subjects: red axis, B-related proteins (miRNA targets) and B genes reported in previous study [11]; green axis, mature miRNAs; blue axis, tissue-specific miRNA targets; orange edges, miRNAs interacting with proteins commonly regulated by miRNAs in all analyzed tissues; yellow edges, miRNAs interacting with tissue-specific proteins; green edges, protein-protein interactions. c Venn diagram of GO terms on B-related proteins and all proteins from predicted A. latifasciata targets

The original Biogrid PPI network contained 544,163 interactions (edges) between 24,913 proteins (nodes). After the experimental detection, redundant edges and species filtering procedures, 294,199 edges between 14, 540 nodes were retained. Based on the 960 B-related proteins whose genes are B-DE-miRNA targets, and B genes identified in a previous study [11], a subnetwork extraction revealed 177 nodes and 335 edges in the Biogrid filtered network. The 177 nodes from the subnetwork are targets of $57 \mathrm{~B}-\mathrm{DE}$-miRNAs from the miRNA: 3'UTR analysis. Finally, all miRNA targets and interactions were added to the subnetwork, resulting in 22,477 edges and 281 nodes (considering both proteins and miRNAs), which was called "B-miR-net" (Fig. $4 \mathrm{~b}$ and Additional File 9).

Despite the ability of B-DE-miRNAs to connect to several mRNAs in B-miR-net, proteins that are regulated in consensus between different tissues are interconnected with each other (Fig. 4b - red axis). Interestingly, among the B-related proteins, connections exist with other proteins that may by indirectly influenced by B-DEmiRNAs (Fig. 4b - edges between red and blue axes). Additionally, 42 of the 102 genes reported on the B chromosome in a previous study [11] are in the B-miRnet, as they are B-DE-miRNA targets or interact with other proteins (Table 2). Nevertheless, 18 of these Bgenes exhibit more than $80 \%$ of integrity in their coding region, meaning potentially translated proteins. The other 24 B-genes displayed less than $80 \%$ of integrity that may generate truncated transcripts what indicates protein translation problems (Table 2 and Additional File 7).

A Gene Ontology (GO) analysis was performed (Additional Files 8), and the proteins were analyzed in the groups of (I) B-related proteins (Fig. 4b - red axis) and (II) all proteins whose genes are targets of $A$. latifasciata miRNAs (Fig. 4b - blue axis). The results revealed 74 biological processes that were shared among the analyzed groups, meaning the common processes between B-related proteins and all A. latifasciata proteins (Fig. 4c, 
Table 2 B-genes present in the B-miR-net. B related proteins - proteins which either are targets of B-DE-miRNAs or interact with these targets. Other proteins: proteins which interact each other and are A. latifasciata miRNA targets

\begin{tabular}{|c|c|c|c|}
\hline & Gene name & Protein Symbol & Integrity (\%) \\
\hline \multirow[t]{11}{*}{ B-related proteins } & apoptosis regulator $\mathrm{Bcl}$-2-like & $\mathrm{BCL} 2$ & 100 \\
\hline & coxsackievirus and adenovirus receptor-like & CXAR & 100 \\
\hline & spindle and kinetochore-associated protein 1-like & SKA1 & 100 \\
\hline & kinesin-like protein KIF11-like & KIF11 & 96.13 \\
\hline & zinc finger protein 771-like & ZNF71 & 89.37 \\
\hline & serine/threonine-protein kinase $\mathrm{RIO}$-like & RIOK3 & 81.57 \\
\hline & ATP-binding cassette sub-family A member 1-like & $\mathrm{ABCA} 1$ & 80.25 \\
\hline & aurora kinase A-B-like & AURKA & 77.16 \\
\hline & centromere-associated protein E-like & CENPE & 73.92 \\
\hline & zinc finger protein 836-like & ZN836 & 64.52 \\
\hline & histone-lysine N-methyltransferase MLL3-like & KMT2C & 56.16 \\
\hline \multirow[t]{31}{*}{ Other proteins } & butyrophilin subfamily 2 member A1-like & $\mathrm{BT} 2 \mathrm{~A} 1$ & 100 \\
\hline & butyrophilin-like protein 2-like & BTNL2 & 100 \\
\hline & myosin-10-like & MYH10 & 100 \\
\hline & polymeric immunoglobulin receptor-like & PIGR & 100 \\
\hline & serine protease 23-like & PRS23 & 100 \\
\hline & peptide chain release factor 1 -like. Mitochondrial-like & RF1ML & 100 \\
\hline & VIP peptides-like & VIP & 100 \\
\hline & zinc finger protein 879-like & ZN879 & 100 \\
\hline & ATP-dependent RNA helicase DDX51-like & DDX51 & 92.22 \\
\hline & CD209 antigen-like & CD209 & 90.13 \\
\hline & protocadherin-10-like & PCD10 & 89.58 \\
\hline & heterogeneous nuclear ribonucleoprotein Q-like & HNRPQ & 79.90 \\
\hline & leucine-rich repeat-containing protein 30 -like & LRC30 & 71.93 \\
\hline & synaptonemal complex protein 2-like & SYCP2 & 70.97 \\
\hline & extracellular calcium-sensing receptor-like & CASR & 69.46 \\
\hline & protein NLRC3-like & NLRC3 & 68.24 \\
\hline & guanine nucleotide-binding protein $\mathrm{G}$ & GNAl1 & 67.52 \\
\hline & vascular cell adhesion protein 1-like & VCAM1 & 65.79 \\
\hline & torsin-1A-interacting protein 2-like & ELOF1 & 64.25 \\
\hline & zinc finger protein 782-like & ZN782 & 63.42 \\
\hline & zinc finger protein 678-like & ZN678 & 63.01 \\
\hline & V-set domain-containing T-cell activation inhibitor 1-like & VTCN1 & 63.00 \\
\hline & poly [ADP-ribose] polymerase 14-like & PAR14 & 61.53 \\
\hline & DNA-directed RNA polymerase E subunit 1-like & RPA49 & 61.01 \\
\hline & endonuclease domain-containing 1 protein-like & ENDD1 & 58.21 \\
\hline & SAM domain and HD domain-containing protein 1-like & ESPL1 & 57.51 \\
\hline & targeting protein for Xklp2-A-like & TPX2 & 52.30 \\
\hline & interferon-induced very large GTPase 1-like & GVIN1 & 51.55 \\
\hline & sterile alpha motif domain-containing protein 12-like & SAM12 & 51.27 \\
\hline & xylulose kinase-like & $X Y L B$ & 50.52 \\
\hline & glucose-6-phosphate 1-dehydrogenase-like & G6PD & 50.43 \\
\hline
\end{tabular}


Additional File 10 - S2). In other words, processes that are commonly present in the cell might be affected by B chromosome presence by miRNA targeting. However, three specific GO terms were found in the B-related protein group, which indicates exclusive processes in the PPI network for genes controlled by the B-DE-miRNAs in all analyzed tissues. These process terms are nuclear matrix organization (GO:0043578), nuclear anchoring at nuclear membrane (GO:0090292), with members of these two terms being the SUN1 (SUN domaincontaining protein 1), SUN2 (SUN domain-containing protein 1) and SYNE1 (syne1) proteins and response to stimulus (GO:0050896), with 141 proteins members (Additional File 9 - S1) (Fig. 4c). Furthermore, a B-gene, ATP-binding cassette sub-family A member 1 (abca1) with $80.25 \%$ of integrity, belongs to response of stimulus biological process. This gene is target of $\mathrm{B}^{+}$upregulated miRNAs in brain, gonad and muscle as describe in the Fig. 5. As highlighted in the Fig. 5a, these two biological processes exclusive of B-related proteins are connected to each other by miRNA targeting.

Furthermore, ABCA1 and SUN2 interact with the proteins CDC42 (cell division control protein 42 homolog) and SKP1 (S-phase kinase associated protein 1), respectively. These two proteins also have GO terms which are common to B-related proteins and all the other proteins. This indicates potential biological processes which occurs in the cells and might be affected by the B chromosome presence. The potential consequences of this interaction under B chromosome presence are developed further in the Discussion section.

\section{Discussion}

In the last few years, B chromosome science has undergone rapid advances due to the wide application of genomics and bioinformatics tools and functional approaches, including analyses of nonmodel species [45]. Here, we advanced the characterization of the miRNome in A. latifasciata, with a focus on the B chromosomes. Initial studies described approximately 200 miRNA genes in fish species [32], and later, this number was increased to 400 genes with the advances in large-scale DNA and RNA sequencing [39]. Here, we identified more than 700 pre-miRNAs, $34 \%$ of which are known miRNAs identified in miRBase (Fig. 1). A set of new premiRNAs in the A. latifasciata genome was described, confirming the rapid evolution of genomes in cichlid species $[32,39,46]$. New miRNAs could arise and be lost quickly mainly between related groups [39]. In this manner, the miRNAs could be tightly involved to the diverse adaptation of Cichlidae family [32, 39]. Some of novel pre-miRNA seeds are similar to cichlid seed sequences, and these new miRNAs are probably isomiRs originated from duplications or as products of RNA editing [43].
The new miRNAs are organized in clusters, which are usually controlled by the same factors to act in the same or related pathways [39]. The A. latifasciata speciesspecific isomiRs might perform new functions related to the B chromosome, as we can observe in the Fig. 5a.

Concerning the investigation of small sequences in the B chromosome, it was not possible to describe a premiRNA in the B extra element based on the adopted approaches. We pointed two reasons: the miRNAs evolution and the limitations of Illumina sequencing to investigated small sequences with low coverage. One of the main reasons for the lack of miRNAs is probably related to the B chromosome structure, which in A. latifasciata originated from a mosaic of duplicated sequences that underwent mutations over time [11]. Even though the difficulty of screening new miRNA sequences in a degenerated element (as the B chromosome), given the limitations of sRNAseq and genomic approaches, we cannot discard to find out small RNAs sequences in the B chromosome. Our results offered a miRNA genomic annotation and expression analysis of a new cichlid fish. This data can improve future studies by association with other genomic sequencing technologies that cover the B chromosome in a better way, such as Pacbio [47] and flow sorting [48].

We observed differences in the expression of miRNAs encoded by the regular chromosomes complement (BDE-miRNAs) in B chromosome carriers. Similar results were also observed in maize [20]. The mechanism by which B chromosomes affect the expression of A complement miRNA genes is unclear. The presence of active genes related to miRNA biogenesis in the $\mathrm{B}$ chromosome might explain these variations. Argonaute (AGO) proteins are essential for processing small RNAs [25, 43], and although AGO-like protein genes have already been observed on the B chromosomes of Secale cereale [49], copies of these genes and other genes related to miRNA biogenesis were not found in A. latifasciata B chromosomes [11, 37]. Moreover, the expression of A copies of drosha and dicer genes, the main regulators of miRNA biogenesis, were not affected by B chromosomes (Additional File 5). Thus, the miRNA pathway does not appear to have changes under the $\mathrm{B}$ chromosome presence.

The B-DE-RNAs are specific to each tissue (Fig. 3), but their targets are usually the same (Fig. 4a). In other words, we have different B-DE-miRNAs targeting common proteins among the tissues and sex. Further, some of these proteins interacts each other in biological processes with relation to B chromosome presence (Fig. 4c). Among the target functions, the cell cycle pathway has attracted attention, since genes related to the cell cycle and chromosome segregation are registered as located on B chromosomes (for a review, see [50]). What would 


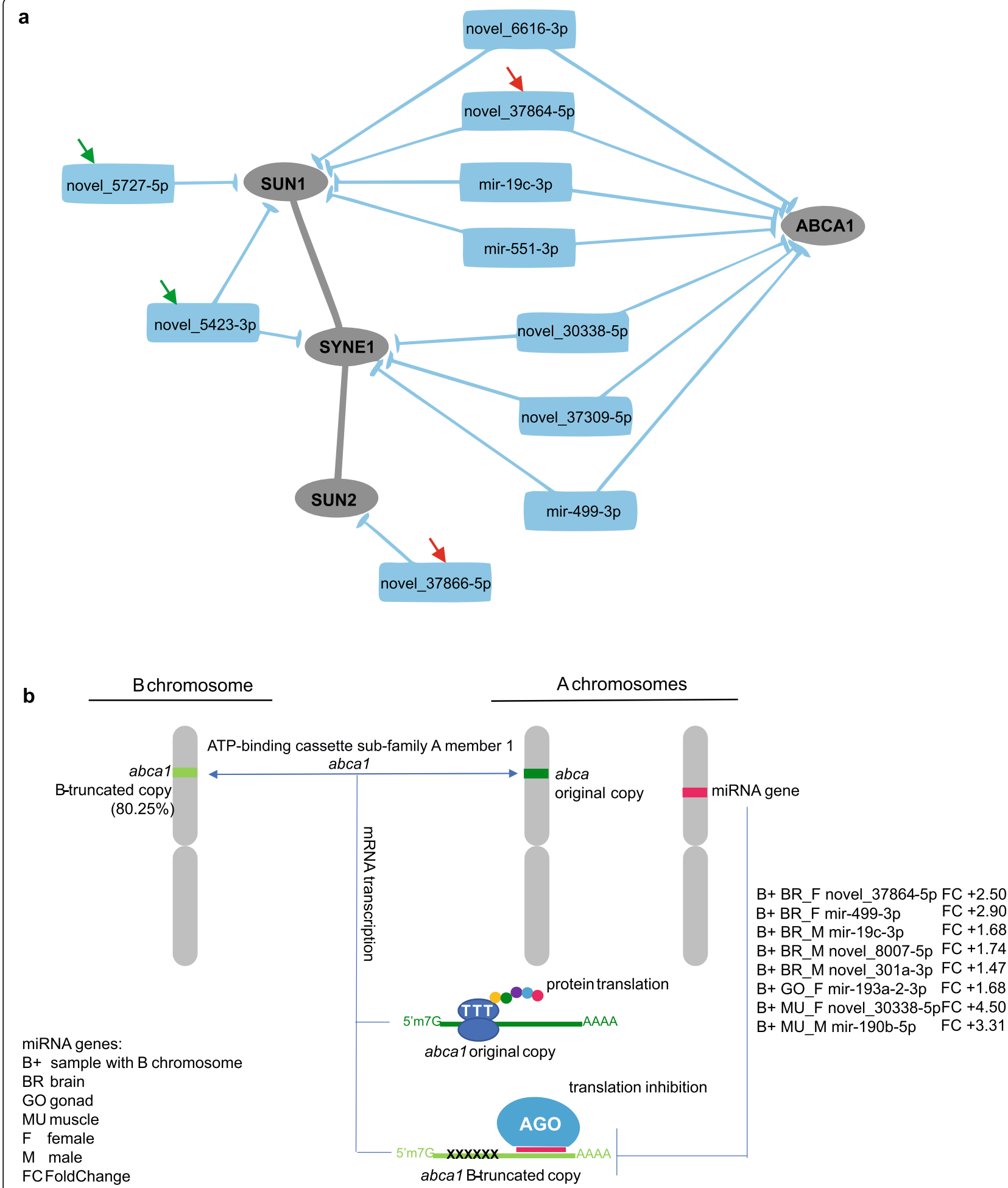

Fig. 5 Potential activity of small RNA genes originated from A chromosomes and their action over the B chromosome. a B-DE-miRNAs targeting the genes of exclusive B-related protein GO terms. The arrows with the same color indicate clustered miRNAs. $\mathbf{b}$ The truncated B-genes of the BmiR-net compete to their original A copy and are controlled by B-DE-miRNAs in the way to benefit B chromosome maintenance 
explain the differential miRNA expression in the presence of the B chromosome? Gene fragments with different degrees of integrity are present in the A. latifasciata B chromosome [11]. We suggest, if these truncated gene copies are transcribed, more binding sites will be available to miRNAs, generating regulatory competition with the A chromosome set (Fig. 5) [51]. We reported a B-gene, abca1, which has $80 \%$ of integrity and is a B-DE-miRNA target (Table 2 and Fig. 5a). Thus, according to the miRNAs controlling these genes, even truncated copies might display increased activity in the cell, further investigations are needed to confirm such assumption. Finally, because the miRNA targets are similar in different tissues, the gene functions affected by their regulation may favor the B chromosome to some extent $[5,14]$.

\section{Conclusions}

We presented the A. latifasciata miRNome and compared it with other available miRNA databases. Additionally, several miRNAs were DE in the brain, gonads and muscle in the $\mathrm{B}^{+}$samples and shared common genes as miRNA targets. The differentially expressed miRNAs detected in the presence of the $\mathrm{B}$ chromosome are not the same among the tissues, but the miRNA targets are involved in the same biological processes. Thus, we suggest that the B chromosome influences the cellular environment using miRNAs as a posttranscriptional control process that is probably for its own benefit of $B$ drive and maintenance.

\section{Materials and methods Samples}

A. latifasciata fishes were obtained from the Integrative Genomics Laboratory fish room at São Paulo State University, Botucatu (SP), Brazil and were genotyped for presence/absence of the $\mathrm{B}$ chromosome using the previously developed marker for $B$ chromosomes [52]. The animals with $\mathrm{B}\left(\mathrm{B}^{+}\right)$and without $\mathrm{B}\left(\mathrm{B}^{-}\right)$were kept in different aquariums until the tissue collection procedure. We used four animals to each group: females $B^{-}$, females $\mathrm{B}^{+}$, males $\mathrm{B}^{-}$and males $\mathrm{B}^{+}$. Twelve animals (four of each group) were used to RNA-seq and twelve to DNA and RNA extraction to PCR, qPCR an RT-qPCR, totalizing 24 animals. The animals were submitted to euthanasia by immersion in eugenol $1 \%$ for $3 \mathrm{~min}$, following the tissue extraction (brain, gonads and muscle) by liquid nitrogen flash frozen method. The tissues were stocked in -80 refrigerators until the RNA and DNA extraction, described below.

\section{RNA sequencing of small RNAs in brain, gonads and muscle}

The total RNA was extracted from the brain and gonads of six males and six females without B chromosomes (B-
) and with $\mathrm{B}$ chromosomes $\left(\mathrm{B}^{+}\right)$and the muscles of four males and four females $\mathrm{B}^{-}$and $\mathrm{B}^{+}$using the TRIzol ${ }^{\mathrm{Ts}}$ (Invitrogen) protocol. The samples were assessed for quality using electrophoresis on an agarose gel and RIN (RNA Integrity Number, >8) parameters and then sent for small RNA sequencing (sRNAseq). This service was executed by Sequencing Service at LC Sciences - Houston, TX, USA, using a single-end Illumina HiSeq 2000 platform after the library construction with $\mathrm{TruSeq}^{\circ}$ Small RNA Sample Preparation (Illumina). The libraries are deposited in the NCBI database (access numbers SRR13040679-SRR13040710).

\section{MicroRNome construction and expression analysis}

The small RNA libraries were filtered for quality using Fastx-Toolkit (http://hannonlab.cshl.edu/fastx_toolkit/ index.html) with the default parameters. Adaptors (TGGAA) and sequences shorter than 17 nucleotides and longer than 27 nucleotides were removed using Cutadapt (https://cutadapt.readthedocs.io/en/stable/) (Additional File 11 - Table S1).

The miRBase fish database [53] (Release 22.1, October 2018) (Additional File 10 - Table S3) was clustered using CD-hit software (default parameters) [54] to create a nonredundant reference list of fish miRNAs.

The filtered small RNA-Seq libraries were used as input for mapping based on default parameters [55] to predict and identify miRNAs (Additional File 11 - Table S2). Then, the nonredundant reference list of fish miRNAs was aligned to the A. latifasciata genome assembly [37] using Bowtie2 (v 2.3.3.1) [56]. The final prediction step consists of the submission of the mapper.pl output, the original nonredundant reference list and the aligned reference list to miRDeep2.0.1.2 analysis using default parameters [55]. The miRDeep software allows identifying known and novel miRNAs based on the existence of the miRNAs in the miRBase. The novel miRNAs were analyzed with BLAST on the seed region (2-8 nucleotides) to characterize if the new miRNA belongs to either a known miRNA family or a known miRNA derived [55].

The differential expression analysis was performed using miaRma pipeline [57] with the parameters desoft = EdgeR-Noiseq; filter $=$ yes; cpmvalue $=1$; repthreshold $=$ 6; fc_threshold $=0.5$; edger_normethod $=\mathrm{TMM}$; replicates $=$ yes; $\quad$ bcvvalue $=0.4 ; \quad$ replicatevalue $=$ biological; and noiseq_normethod $=\mathrm{tmm}$. The comparisons were performed separately for each tissue (brain, gonads and muscle), sex (male and female) and the presence or absence of the $\mathrm{B}$ chromosome $\left(\mathrm{B}^{+} / \mathrm{B}^{-}\right)$, always considering the $\mathrm{B}$ - as control. The miRNAs with $p<0.05$ were considered differentially expressed (DE).

\section{Analysis of B chromosome copies}

Three strategies were adopted to identify possible copies of miRNA genes related to the B chromosome. The first 
was based on "coverage ratio analysis" [11]. The miRNA annotations of the A. latifasciata genome were compared to the $\mathrm{B}^{-}$and $\mathrm{B}^{+}$read coverage ratios of the $A$. latifasciata genome. The regions with twice as high coverage read in the $\mathrm{B}+$ genome indicate a duplicated $\mathrm{B}$ chromosome sequence copy [11] (i.e.: $60 x$ coverage $B^{-}$ reads and 120x coverage $B^{+}$reads). The second strategy was to use all miRNA reads (from $\mathrm{B}^{-}$and $\mathrm{B}^{+}$samples) that failed to align the $A$. latifasciata genome during miRNome construction to identify $B$ chromosomespecific miRNAs. The unaligned reads were used for miRNA identification by miRDeep using the $\mathrm{B}^{+}$genome assembly [37], followed by the prediction of miRNA: mRNA interactions by TargetScan (described further below). To analyze if these sequences are exclusive from B chromosome, we performed a BLAST search using Metriaclima zebra genome (v0, bouillabase.org, last accessed on October 16, 2020). The scaffold matched from $M$. zebra genome was visualized with $A$. latifasciata $\mathrm{B}^{-}$and $\mathrm{B}^{+}$genomic read alignments on Saci Base Jbrowser (https://sacibase.ibb.unesp.br/index.html, last accessed on October 16, 2020). Finally, the third strategy was based on the duplicated B chromosome blocks (Bblocks) identified in a previous study [37]. The previously predicted miRNAs located on B-blocks were used to predict the miRNA:mRNA interactions with TargetScan (described further below).

\section{3'UTR prediction and miRNA:3'UTR interactions}

The 3'UTR sequences from the A. latifasciata transcriptome [58] were predicted using Transdecoder (v5.5.0) [59]. The annotation file for each transcript was used with Bedtools (v2.26.2) [60] to retrieve the predicted 3'UTR for further analyses of interactions. Additionally, the transcript tissue specificity was retrieved based on observed expression [58] to assess tissue-specific interactions.

The miRNAs (novel and known) identified on miRNome and the 3'UTR from the A. latifasciata transcriptome were submitted to TargetScan 6.0 [42] to predict the miRNA:3'UTR interactions. Scores less than -0.2 were considered significant predictions in accordance with software parameters.

\section{Genomic PCR, qPCR and RT-qPCR}

Genomic DNA was extracted using [61] protocol. The samples were genotyped using the molecular marker for the presence of the B chromosomes [52]. Primers for mature miRNA sequences were constructed using Primer3Plus. Conventional genomic PCR was performed using Taq DNA Polymerase (Invitrogen-10,342-053) submitted to cycling at $94{ }^{\circ} \mathrm{C}$ for $5 \mathrm{~min}$, followed by 35 cycles $\left(94{ }^{\circ} \mathrm{C}\right.$ for $1 \mathrm{~min}, 50^{\circ} \mathrm{C}$ to $60^{\circ} \mathrm{C}$ for $45 \mathrm{~s}$, and $72{ }^{\circ} \mathrm{C}$ for $10 \mathrm{~min}$ ) and $72^{\circ} \mathrm{C}$ for $10 \mathrm{~min}$. The result was verified by agarose gel electrophoresis (1\%). The genomic DNA of eight $\mathrm{B}^{-}$and eight $\mathrm{B}^{+}$samples, including samples from males and females, was used for GDR analysis with qPCR and SYBR Green detection (Ampliqon) to verify the putative miRNAs associated with the $\mathrm{B}$ chromosome copy. The gene expression analysis was based on the total RNA extracted from the brain, gonads and muscle of $\mathrm{B}^{-}$and $\mathrm{B}^{+}$of both sexes, and performed in biological triplicates. The samples were converted to cDNA libraries (High-Capacity RNA-to-cDNA ${ }^{\mathrm{m}}$ Kit, Applied Biosystems) and used for RT-qPCR with SYBR Green detection (Ampliqon). The $\Delta \Delta \mathrm{C}_{\mathrm{q}}$ method was used to analyze relative expression [62]. Q-Gene software [63] was used for normalization with the ubiquitin-conjugating enzyme (UBCE) as the reference gene. The primers are described in Additional File 11 - Table S4.

\section{Network construction and functional analysis}

The human PPI network was downloaded from Biogrid to analyze miRNA functions [64]. The network was filtered to retain only nonredundant interactions between human proteins detected by affinity chromatography technology (MI: 0004), X-ray crystallography (MI:0114), far western blotting (MI:0047), fluorescent resonance energy transfer (MI:0055), protein complementation assay (MI:0090), experimental interaction detection (MI:0045) and two hybrid (MI:0018) experiments. The annotated protein-coding transcripts [58] commonly regulated by B-DE-miRNAs in each tissue (brain, gonads and muscle), together with $B$ genes reported in a previous study [11], were used to extract a PPI subnetwork from the filtered Biogrid. The miRNA:3'UTR interactions were added to the PPI subnetwork to create the final B-miR-net. The protein list was subjected to a Gene Ontology (GO) analysis using gProfiler to verify exclusive and specific GO terms for proteins derived from B-DE-miRNA targets and the remaining proteins from all $A$. latifasciata targets [65].

\section{Abbreviations \\ 3'UTR: $3^{\prime}$ untranslated region; $B^{-}$: Samples without B chromosome; $\mathrm{B}^{+}$: Samples with $\mathrm{B}$ chromosome; B-blocks: $\mathrm{B}$ chromosome sequences; B-DE- miRNAs: Differentially expressed miRNAs in the $\mathrm{B}^{+}$samples; $\mathrm{B}$-miR- net: Protein-protein and B-DE-miRNAs interaction network; $C_{a}$ : Quantification cycle; DE miRNAs: Differentially expressed microRNAs; GDR: Gene dose ratio; GO: Gene Ontology; miRNA:mRNA: microRNA and messenger RNA interaction; miRNAs: microRNAs; mRNAs: Messenger RNA; PCR: Polymerase chain reaction; piRNAs: PIWI-Interacting RNAs; PPI: Protein-protein interaction; pre-miRNA: Precursor microRNA; pri-miRNA: Primary microRNA; PSR: Paternal sex ratio; qPCR: Quantitative real-time PCR; RIN: RNA Integrity Number; RNA- seq: RNA sequencing; RT-qPCR: Reverse transcription-qPCR; siRNAs: Small interference RNAs}

\section{Supplementary Information}

The online version contains supplementary material available at https://doi. org/10.1186/s12864-021-07651-w.

Additional file 1. miRDeep2 - Astatotilapia latifasciata miRNA prediction. 
Additional file 2. Html file of miRNA prediction. miRDeep2 - miRNA prediction in the $B^{+}$assembly using $B^{-}$RNAseq which did not align in the $A$. latifasciata genome.

Additional file 3. $\mathrm{Html}$ file of miRNA prediction. miRDeep2 - miRNA prediction in the $\mathrm{B}^{+}$assembly using $\mathrm{B}^{+}$RNAseq which did not align in the $A$. latifasciata genome.

Additional file 4. Nucleotide sequences (Figure S1) and reads coverage (Figure S2) of pre-miRNAs sequences on the $\mathrm{B}^{+}$assembled genome. Figure S1. Sequence descriptions: \#primer $>$ NODE contigXXXX Mzebra scaffold_XX start:end $\mathrm{B}^{-} / \mathrm{B}^{+}$reads. The primer binding sites are highlighted in bold. Figure S2. Genome assembly bias. The red rectangles on the graphs highlight the pre-miRNAs regions in the $M$. zebra genome and $A$. latifascita $\mathrm{B}^{-}$and $\mathrm{B}^{+}$genomic reads datasets. These miRNAs were predicted in the $\mathrm{B}^{+}$assembly using the reads that failed to align in the $\mathrm{B}^{-}$ reference assembly expecting to find some $B^{+}$chromosome miRNA.

Additional file 5. Expression of drosha and dicer genes.

Additional file 6. Html files containing miRNA prediction in B-blocks, separated by RNA-seq sample. S1 miRDeep2 - miRNA prediction in Bblocks using brain female $B^{-}$RNA-seq. $\mathbf{S 2}$ miRDeep2 - miRNA prediction in B-blocks using brain female $B^{+}$RNA-seq. S3 miRDeep2 - miRNA prediction in B-blocks using brain male $B^{-}$RNA-seq. $\mathbf{S 4}$ miRDeep2 - miRNA prediction in B-blocks using brain male $B^{+}$RNA-seq. S5 miRDeep2 miRNA prediction in B-blocks using gonads female $B^{-}$RNA-seq. S6 miRDeep2 - miRNA prediction in B-blocks using gonads female $B^{+}$RNA-seq. S7 miRDeep2 - miRNA prediction in B-blocks using gonads male B RNA-seq. $\mathbf{S 8}$ miRDeep2 - miRNA prediction in B-blocks using gonads male RNA-seq. $\mathbf{S 9}$ miRDeep2 - miRNA prediction in B-blocks using muscle female $B^{-}$RNA-seq. S10 miRDeep2 - miRNA prediction in Bblocks using muscle female $B^{+}$RNA-seq. $\mathbf{S 1 1}$ miRDeep2 - miRNA prediction in B-blocks using muscle male B ${ }^{-}$RNA-seq. S12 miRDeep2 - miRNA prediction in B-blocks using muscle male $B^{+}$RNA-seq.

Additional file 7. Multiple data sheets of samples separated by tissue and sex describing the B-DE-miRNA and its target.

Additional file $\mathbf{8}$. Multiple data sheets of samples separated by tissue and sex describing the B-DE miRNA target gene and protein names - recovered from A. latifasciata transcriptome annotation [58]

Additional file 9. Folder containing the tsv files of networks. S1.tsv PPI network filtered from the Biogrid database. S2.tsv PPI of all proteins from A. latifasciata miRNA targets. S3.tsv B-related proteins and B genes subnetwork. S4.tsv B-miR-net.

Additional file 10. $\mathrm{GO}$ datasets. S1 Exclusive $\mathrm{GO}$ terms for proteins derived from B-DE-miRNA targets and B genes. S2 Common GO terms among B-related proteins and other proteins. S3 GO terms for all A. latifasciata targets in the PPI network.

Additional file 11. Samples and primers information. Table S1. RNAseq quality and filtering. Table S2. Normalized reads mapped in the genome. Table S3. miRBase fish database entries used to create the reference miRNA list. Table S4. Primers used for genomic PCR, genomic qPCR and RT-qPCR.

\section{Authors' contributions}

$J I N, B E A F$ and CM designed the study. JIN, BEAF, ALC and ER collaborated to perform the experiments. JIN, IRW and NR contributed to bioinformatics approaches. RO constructed a statistical method for this study based on the data distribution and $\mathrm{B}$ chromosome information. JIN analyzed the results and wrote the paper with approval from all authors.

\section{Funding}

This research was financially supported by grants from the São Paulo Research Foundation (FAPESP) - grant numbers 2015/16661-1, 2016/093206, and 2017/25193-7, and the National Council of Technological and Scientific Development (CNPq) - grant number 304111/2020-1.

\section{Availability of data and materials}

The A. latifasciata $\mathrm{B}^{+}$assemblies and B-blocks are available in a previous study under Bioproject PRJN369442 access [37] and can be visualized on https://sacibase.ibb.unesp.br/. The A. latifasciata miRNAs sequences are shown in Additional Files. The targets sequences have been published [58] The small RNA-seq libraries are deposited in the NCBI database (accession numbers from SRR13040679 to SRR13040710b).

\section{Declarations}

\section{Ethics approval and consent to participate}

The experiments involving all animals were performed according to the agreement of ethics set by the Brazilian College of Animal Experimentation and the ARRIVE (Animal Research: Reporting In-vivo Experiments) guidelines, and the use of all specimens in the experiments was approved by the ethics committee of the Institute of Biosciences/Unesp (Protocol no. 769-2015).

\section{Consent for publication}

Not applicable.

\section{Competing interests}

The authors have no competing interests to declare.

\section{Author details}

${ }^{1}$ Department of Structural and Functional Biology, Institute of Bioscience at Botucatu, São Paulo State University (UNESP), Botucatu, SP 18618-689, Brazil.

${ }^{2}$ Medical School, University of Ninth of July (UNINOVE), Bauru, SP, Brazil. ${ }^{3}$ Faculty of Mathematics and Natural Sciences, University of Bonn, Bonn, Germany. ${ }^{4}$ Department of Biostatistics, Plant Biology, Parasitology and Zoology, Institute of Bioscience at Botucatu, São Paulo State University (UNESP), Botucatu, SP, Brazil.

Received: 19 January 2021 Accepted: 23 April 2021

Published online: 12 May 2021

\section{References}

1. Jones RN. B-chromosome drive. Am Nat. 1991;137(3):430-42. https://doi. org/10.1086/285175

2. Miao VP, Covert SF, Vanetten HD. A fungal gene for antibiotic resistance on a dispensable ("B") chromosome. Science. 1991;254(5039):1773-6. https:// doi.org/10.1126/science.1763326.

3. Camacho JPM, Sharbel TF, Beukeboom LW. B-chromosome evolution. PhilTrans R Soc Lond. 2000;355(1394):163-78. https://doi.org/10.1098/rstb.2 000.0556

4. Jones RN, González-Sánchez M, González-García M, Vega JM, Puertas MJ. Chromosomes with a life of their own. Cytogenet Genome Res. 2008;120(34):265-80. https://doi.org/10.1159/000121076.

5. Houben A, Banaei-Moghaddam AM, Klemme S, Timmis JN. Evolution and biology of supernumerary B chromosomes. Cell Mol Life Sci. 2014;71(3):46778. https://doi.org/10.1007/s00018-013-1437-7.

6. Ploskaya-Chaibi M, Voitovich AM, Novitsky RV, Bouhadad R. B-chromosome and $\mathrm{V}$-shaped spot asymmetry in the common frog (Rana temporaria $\mathrm{L}$ ) populations. Comptes Rendus - Biol. 2015;338(3):161-8. https://doi.org/10.1 016/j.crvi.2014.12.005.

7. Bueno D, Palacios-Gimenez OM, Cabral-de-Mello DC. Chromosomal mapping of repetitive DNAs in the grasshopper Abracris flavolineata revea possible ancestry of the $\mathrm{B}$ chromosome and $\mathrm{H} 3$ histone spreading. PLoS One. 2013;8(6):e66532.

8. De Silva DMZA, Utsunomia R, Ruiz-Ruano FJ, Daniel SN, Porto-Foresti F Hashimoto DT, et al. High-throughput analysis unveils a highly shared satellite DNA library among three species of fish genus Astyanax. Sci Rep. $2017 ; 7(1): 1-12$.

9. Coan RLB, Martins C. Landscape of transposable elements focusing on the chromosome of the cichlid fish Astatotilapia latifasciata. Genes (Basel). 2018; 9(6):269.

10. Graphodatsky AS, Kukekova AV, Yudkin DV, Trifonov VA, Vorobieva NV, Beklemisheva VR, et al. The proto-oncogene C-KIT maps to canid Bchromosomes. Chromosom Res. 2005;13(2):113-22. https://doi.org/10.1007/ s10577-005-7474-9.

11. Valente GT, Conte MA, Fantinatti BEA, Cabral-De-Mello DC, Carvalho RF, Vicari MR, et al. Origin and evolution of B chromosomes in the cichlid fish Astatotilapia latifasciata based on integrated genomic analyses. Mol Biol Evol. 2014;31(8):2061-72. https://doi.org/10.1093/molbev/msu148.

12. Rajičić M, Adnadević T, Stamenković G, Blagojević J, Vujošević M. Screening of $\mathrm{B}$ chromosomes for presence of two genes in yellow-necked mice, 
Apodemus flavicollis (Mammalia, Rodentia). Genetika. 2015;47(1):311-21. https://doi.org/10.2298/GENSR1501311R.

13. Navarro-Domínguez B, Ruiz-Ruano FJ, Cabrero J, Corral JM, López-León MD, Sharbel TF, et al. Protein-coding genes in B chromosomes of the grasshopper Eyprepocnemis plorans. Sci Rep. 2017;7(September 2016):1-12. Available from:. https://doi.org/10.1038/srep45200.

14. Banaei-Moghaddam AM, Meier K, Karimi-Ashtiyani R, Houben A. Formation and expression of pseudogenes on the $B$ chromosome of rye. Plant Cell. 2013;25(7):2536-44 Available from: http://www.plantcell.org/cgi/doi/10.11 05/tpc.113.111856.

15. Carmello BO, Coan RLB, Cardoso AL, Ramos E, Fantinatti BEA, Marques DF, et al. The hnRNP Q-like gene is retroinserted into the B chromosomes of the cichlid fish Astatotilapia latifasciata. Chromosom Res. 2017;25(3-4):27790. https://doi.org/10.1007/s10577-017-9561-0.

16. Martis MM, Klemme S, Banaei-Moghaddam AM, Blattner FR, Macas J, Schmutzer T, et al. Selfish supernumerary chromosome reveals its origin as a mosaic of host genome and organellar sequences. Proc Natl Acad Sci. 2012;109(33):13343-6 Available from: http://www.pnas.org/cgi/doi/10.1073/ pnas.1204237109.

17. Akbari OS, Antoshechkin I, Hay BA, Ferree PM. Transcriptome profiling of Nasonia vitripennis pestis reveals novel transcripts expressed from the selfish B chromosome, paternal sex ratio. G3; Genes|Genomes|Genetics. 2013;3(9): 1597-605 Available from: http://g3journal.org/lookup/doi/10.1534/g3.113. 007583.

18. Li Y, Jing XA, Aldrich JC, Clifford C, Chen J, Akbari OS, et al. Unique sequence organization and small RNA expression of a "selfish" B chromosome. Chromosoma. 2017;126(6):753-68. https://doi.org/10.1007/ s00412-017-0641-X.

19. Ramos É, Cardoso AL, Brown J, Marques DF, Fantinatti BEA, Cabral-de-Mello $D C$, et al. The repetitive DNA element BncDNA, enriched in the $B$ chromosome of the cichlid fish Astatotilapia latifasciata, transcribes a potentially noncoding RNA. Chromosoma. 2017;126(2):313-23. Available from: https://doi.org/10.1007/s00412-016-0601-X.

20. Huang YH, Peng SF, Lin YP, Cheng YM. The maize B chromosome is capable of expressing microRNAs and altering the expression of microRNAs derived from a chromosomes. Chromosom Res. 2020;28(2):129-38. https:// doi.org/10.1007/s10577-019-09620-2.

21. Valente GT, Nakajima RT, Fantinatti BEA, Marques DF, Almeida RO, Simões $\mathrm{RP}$, et al. B chromosomes: from cytogenetics to systems biology. Chromosoma. 2017;126(1):73-81. Available from:. https://doi.org/10.1007/ s00412-016-0613-6.

22. Ahmad $\mathrm{S}$, Martins $\mathrm{C}$. The modern view of $B$ chromosomes under the impact of high scale omics analyses. Cells. 2019;8(2):156. https://doi.org/10.3390/ cells8020156.

23. Huang $Y$, Peng $S$, Lin $Y$. The maize $B$ chromosome is capable of expressing microRNAs and altering the expression of microRNAs derived from a chromosomes. Chrom Res. 2020;28(2):129-38. https://doi.org/10.1007/s1 0577-019-09620-2.

24. Grün D, Wang YL, Langenberger D, Gunsalus KC, Rajewsky N. MicroRNA target predictions across seven Drosophila species and comparison to mammalian targets. PLoS Comput Biol. 2005;1(1):0051-66.

25. Moazed D. Small RNAs in transcriptional gene silencing and genome defence. Nature. 2009:457(7228):413-20. https://doi.org/10.1038/na ture07756

26. Budak H, Zhang B. MicroRNAs in model and complex organisms. Funct Integr Genomics. 2017;17(2-3):121-4. https://doi.org/10.1007/s10142-017-0544-1.

27. Griffiths-Jones S, Hui JHL, Marco A, Ronshaugen M. MicroRNA evolution by arm switching. EMBO Rep. 2011;12(2):172-7. Available from: https://doi. org/10.1038/embor.2010.191.

28. Leung AKL, Sharp PA. MicroRNA functions in stress responses. Mol Cell. 2010; 40(2):205-15. Available from:. https://doi.org/10.1016/.molcel.2010.09.027.

29. D'Ambrosio U, Alonso-Lifante MP, Barros K, Kovařík A, Mas de Xaxars G, Garcia S. B-chrom: A database on B-chromosomes of plants, animals and fungi. New Phytol. 2017;216:635.

30. Kaufman LS, Chapman L, Chapman CA. Evolution in fast forward: Haplochromine fishes of the Lake Victoria region. Endeavour. 1997;21 (1):2330. https://doi.org/10.1016/S0160-9327(96)10034-X.

31. Turner GF. Adaptive radiation of cichlid fish. Curr Biol. 2007:17(19):827-31.

32. Brawand D, Wagner CE, Li YI, Malinsky M, Keller I, Fan S, et al. The genomic substrate for adaptive radiation in African cichlid fish. Nature. 2015; 513(7518):375-81.
33. Poletto $A B$, Ferreira IA, Martins $C$. The $B$ chromosomes of the African cichlid fish Haplochromis obliquidens harbour 185 rRNA gene copies. BMC Genet. 2010;11(1):1-8. https://doi.org/10.1186/1471-2156-11-1.

34. Martins $C$, Jehangir $M$. A genomic glimpse of $B$ chromosomes in cichlids. Genes Genomics. 2021;43(3):199-208. https://doi.org/10.1007/s13258-021-01 049-4.

35. Fantinatti BEAA, Mazzuchelli J, Martins C, Valente GT, Cabral-de-Mello DC, Martins C. Genomic content and new insights on the origin of the B chromosome of the cichlid fish Astatotilapia latifasciata. Genetica. 2011; 139(10):1273-82. https://doi.org/10.1007/s10709-012-9629-x.

36. Cardoso AL, Fantinatti BE de A, Venturelli NB, Carmello B de O, de RA O, Martins C. Epigenetic DNA modifications are correlated with B chromosomes and sex in the cichlid Astatotilapia latifasciata. Front Genet. 2019;10:1-14.

37. Jehangir M, Ahmad SF, Cardoso AL, Ramos E, Valente GT, Martins C. De novo genome assembly of the cichlid fish Astatotilapia latifasciata reveals a higher level of genomic polymorphism and genes related to $B$ chromosomes. Chromosoma. 2019;128(2):81-96. https://doi.org/10.1007/ s00412-019-00707-7.

38. Navarro-Domínguez B, Martín-Peciña M, Ruiz-Ruano FJ, Cabrero J, Corral JM, López-León MD, et al. Gene expression changes elicited by a parasitic B chromosome in the grasshopper Eyprepocnemis plorans are consistent with its phenotypic effects. Chromosoma. 2019;128(1):53-67. https://doi.org/10.1 007/s00412-018-00689-y.

39. Xiong P, Schneider RF, Hulsey CD, Meyer A, Franchini P. Conservation and novelty in the microRNA genomic landscape of hyperdiverse cichlid fishes. Sci Rep. 2019;9(1):1-12. Available from:. https://doi.org/10.1038/s41598-01950124-0.

40. Friedländer MR, Chen W, Adamidi C, Maaskola J, Einspanier R, Knespel S, et al. Discovering microRNAs from deep sequencing data using miRDeep. Nat Biotechnol. 2008;26(4):407-15. https://doi.org/10.1038/nbt1394.

41. Oliveira AC, Bovolenta LA, Nachtigall PG, Herkenhoff ME, Lemke N, Pinhal D. Combining results from distinct microRNA target prediction tools enhances the performance of analyses. Front Genet. 2017:8(MAY):1-10.

42. Ulitsky I, Shkumatava A, Jan CH, Subtelny AO, Koppstein D, Bell GW, et al. Extensive alternative polyadenylation during zebrafish development Genome Res. 2012;22(10):2054-66. https://doi.org/10.1101/gr.139733.112.

43. Bartel DP. Metazoan MicroRNAs. Cell. 2018;173(1):20-51. Available from. https://doi.org/10.1016/j.cell.2018.03.006.

44. Rajewsky N. Microrna target predictions in animals. Nat Genet. 2006;38(6S): S8-13. https://doi.org/10.1038/ng1798.

45. Ruban A, Schmutzer T, Scholz U, Houben A. How next-generation sequencing has aided our understanding of the sequence composition and origin of B chromosomes. Genes (Basel). 2017:8(11):1-14

46. Franchini $P$, Xiong P, Fruciano C, Schneider RF, Woltering JM, Hulsey CD, et al. MicroRNA gene regulation in extremely young and parallel adaptive radiations of crater lake cichlid fish. Mol Biol Evol. 2019;36(11):2498-511. https://doi.org/10.1093/molbev/msz168.

47. Clark FE, Conte MA, Kocher TD. Genomic characterization of a B chromosome in lake Malawi cichlid fishes. Genes (Basel). 2018;9(12):610.

48. Karafiátová M, Bedná M, Said M, Jana Č, Blavet N, Bartoš J. The B chromosome of Sorghum purpureosericeum reveals the first pieces of its sequence. J Exp Bot. 2020;72(5):1606-16.

49. Ma W, Gabriel TS, Martis MM, Gursinsky T, Schubert V, Vrána J, et al. Rye B chromosomes encode a functional Argonaute-like protein with in vitro slicer activities similar to its a chromosome paralog. New Phytol. 2017; 213(2):916-28. https://doi.org/10.1111/nph.14110.

50. Benetta ED, Akbari OS, Ferree PM. Sequence expression of supernumerary $B$ chromosomes: Function or fluff? Genes (Basel). 2019;10(2):123.

51. Banaei-moghaddam AM, Martis MM, Gundlach H, Himmelbach A, Altschmied L, Mayer KFXX, et al. Biochimica et Biophysica Acta genes on B chromosomes : old questions revisited with new tools. Biochim Biophys Acta - Gene Regul Mech. 2015;1849(1):64-70. https://doi.org/10.1016/j.bba grm.2014.11.007.

52. Fantinatti BEAA, Martins C. Development of chromosomal markers based on next-generation sequencing: the $B$ chromosome of the cichlid fish Astatotilapia latifasciata as a model. BMC Genet. 2016;17(1):1-8. Available from:: https://doi.org/10.1186/s12863-016-0427-9.

53. Kozomara A, Birgaoanu M, Griffiths-Jones S. MiRBase: from microRNA sequences to function. Nucleic Acids Res. 2019:47(D1):D155-62. https://doi. org/10.1093/nar/gky1141. 
54. Li W, Godzik A. Cd-hit: a fast program for clustering and comparing large sets of protein or nucleotide sequences. Bioinformatics. 2006;22(13):1658-9. https://doi.org/10.1093/bioinformatics/bt1158.

55. Friedländer MR, Mackowiak SD, Li N, Chen W, Rajewsky N. MiRDeep2 accurately identifies known and hundreds of novel microRNA genes in seven animal clades. Nucleic Acids Res. 2012;40(1):37-52. https://doi.org/10.1 093/nar/gkr688.

56. Langmead B, Salzberg SL. Fast gapped-read alignment with bowtie 2. Nat Methods. 2012;9(4):357-9. https://doi.org/10.1038/nmeth.1923.

57. Andrés-León E, Núñez-Torres R, Rojas AM. miARma-Seq: a comprehensive tool for miRNA, mRNA and circRNA analysis. Sci Rep. 2016;6:1-8.

58. Nakajima RT. Análises em larga escala de proteínas e construção de redes biológicas com foco em estudos de cromossomos B; 2019. p. 1-20.

59. Haas BJ, Papanicolaou A, Yassour M, Grabherr M, Blood PD, Bowden J, et al. De novo transcript sequence reconstruction from RNA-seq using the trinity platform for reference generation and analysis. Nat Protoc. 2013;8(8):1494512. https://doi.org/10.1038/nprot.2013.084.

60. Quinlan AR, Hall IM. BEDTools: a flexible suite of utilities for comparing genomic features. Bioinformatics. 2010;26(6):841-2. https://doi.org/10.1093/ bioinformatics/bta033.

61. Sambrook J, Russell DW. Purification of nucleic acids by extraction with phenol:chloroform. Cold Spring Harb Protoc. 2006;2006(1):pdb.prot4455.

62. de Santis C, Smith-Keune C, Jerry DR. Normalizing RT-qPCR data: are we getting the right answers? An appraisal of normalization approaches and internal reference genes from a case study in the finfish Lates calcarifer. Mar Biotechnol. 2011;13(2):170-80. https://doi.org/10.1007/s10126-010-9277-z.

63. Simon P. Q-Gene: Processing quantitative real-time RT-PCR data. Bioinformatics. 2003;19(11):1439-40. https://doi.org/10.1093/bioinformatics/ btg157.

64. Chatr-Aryamontri A, Breitkreutz BJ, Oughtred R, Boucher L, Heinicke S, Chen D, et al. The BioGRID interaction database: 2015 update. Nucleic Acids Res. 2015;43(D1):D470-8. https://doi.org/10.1093/nar/gku1204.

65. Raudvere U, Kolberg L, Kuzmin I, Arak T, Adler P, Peterson H, et al. G:profiler: a web server for functional enrichment analysis and conversions of gene lists (2019 update). Nucleic Acids Res. 2019;47(W1):W191-8. https://doi.org/1 0.1093/nar/gkz369.

\section{Publisher's Note}

Springer Nature remains neutral with regard to jurisdictional claims in published maps and institutional affiliations.

Ready to submit your research? Choose BMC and benefit from:

- fast, convenient online submission

- thorough peer review by experienced researchers in your field

- rapid publication on acceptance

- support for research data, including large and complex data types

- gold Open Access which fosters wider collaboration and increased citations

- maximum visibility for your research: over $100 \mathrm{M}$ website views per year

At $\mathrm{BMC}$, research is always in progress.

Learn more biomedcentral.com/submissions 